\title{
Regular pulse rate but irregular heart rate?
}

\author{
B. Bellmann ${ }^{1,4} \cdot$ C. Gemein ${ }^{2}$ P. Schauerte ${ }^{3}$
}

The 12-lead ECG shows a ventricular bigeminy with right bundle branch block, which is evidence of a left ventricular origin. The inferior axis and repetitive monomorphic occurrence points to an origin from the outflow tract. Thus, the diagnosis is repetitive left ventricular outflow tract premature beats. This extrasystole can be found in healthy individuals and does not increase the risk of sudden cardiac death. However, due to the short coupling interval of the extrasystole there is almost no diastolic filling before the extrasystolic beat, thus leading to a pulse deficit which is aggravated by the lack of a subsequent sinus beat (postextrasystolic pause). The subsequent sinus beat is augmented due to postextrasystolic potentiation and a prolonged diastole. During bigeminy there is a 2:1 pulse deficit leading to the reported slow but regular pulse rates of the patient. Outflow tract premature beats often show a fixed coupling interval due to triggered activity during phase III of the action potential (early afterdepolarisation) and thus sometimes responds to calcium channel inhibitors such as verapamil. Beta-blockers may be effective but sides effects such

\section{B. Bellmann}

barbara.bellmann@charite.de

1 Department of Cardiology, Charité Berlin Campus Benjamin Franklin, Berlin, Germany

2 Department of Cardiology, University Hospital Gießen, Gießen, Germany

3 Kardiologie an der Rudower Chaussee, Berlin, Germany

4 Department of Cardiology, University Hospital, Technical University Aachen RWTH,

Aachen, Germany

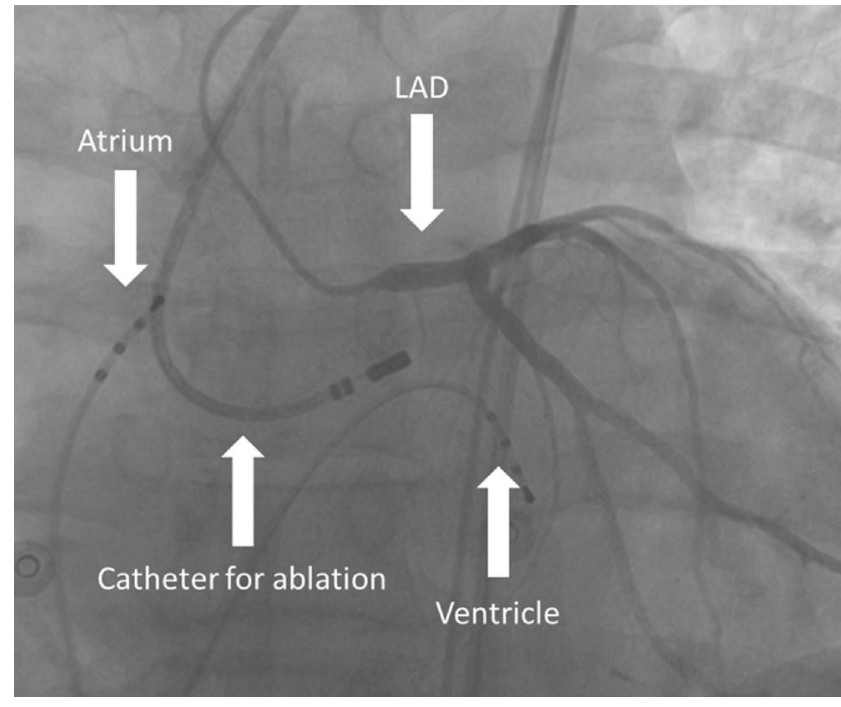

Fig. 1 Ablation catheter in the ascending aorta below the left main coronary artery as visualised by a diagnostic angiography catheter (JL-4). Additional electrode catheters are positioned in the high right atrium and right ventricle. Due to the anatomical proximity of the coronary artery to the origin of the arrhythmia at the left coronary sinus cusp, ablation is carried out under imaging of the coronary vessels. The ablation catheter and two diagnostic catheters, one in the atrium and one in the ventricle, are also shown. (LAD left anterior descending)

as arterial hypotension decrease compliance, especially in young adults.

Accordingly, in this patient, ablation of the arrhythmia was scheduled. For electrophysiological mapping, a deflectable mapping and ablation catheter was introduced retrogradely into the left ventricle and mapping of the earliest ventricular activity was performed during spontaneous ventricular premature beats. In this case, earliest activity was recorded above the aortic valve inside the left coronary sinus cusp. Pacing from this site revealed a similar 


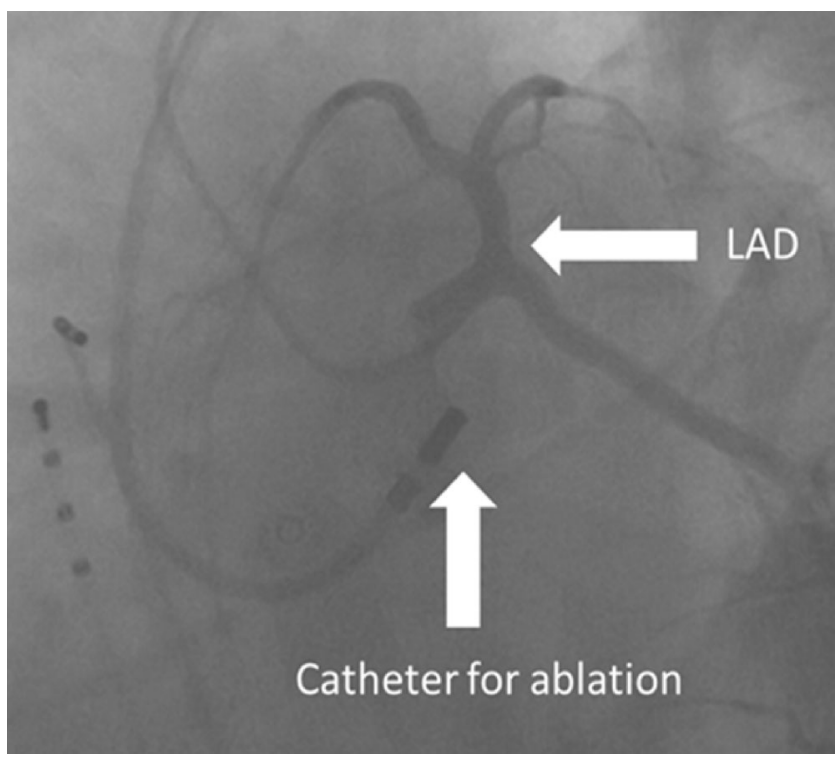

Fig. 2 Under such ablations at critical points, imaging from different levels is important to safely prevent injury to the coronary arteries. ( $L A D$ left anterior descending)

12-lead ECG morphology as during spontaneous ventricular premature beats. Due to the proximity to the left main coronary artery, a left coronary angiography catheter was positioned into the left main coronary artery (Fig. 1 and 2) and simultaneous visualisation during ablation at this site was performed to identify impeding damage to the coronary vessel [1]. Ablation was performed using an irrigated catheter and the arrhythmia terminated after 10 seconds of radiofrequency ablation (Fig. 3). On the right side of the ECG you can see ST elevation which is documented near the coronary artery. After the ablation, this completely resolved. Since then, the patient has been free of symptoms and repetitive Holter ECGs did not show a recurrence of the arrhythmia.

Conflict of interest B. Bellmann, C. Gemein and P. Schauerte state that there are no conflicts of interest.

\section{References}

1. Jauregui AME, Campos B, Park KM, et al. Ablation of ventricular arrhythmias arising near the anterior epicardial veins from the left sinus of Valsalva region: ECG features, anatomic distance, and outcome. Heart Rhythm. 2012;9:865-873. 


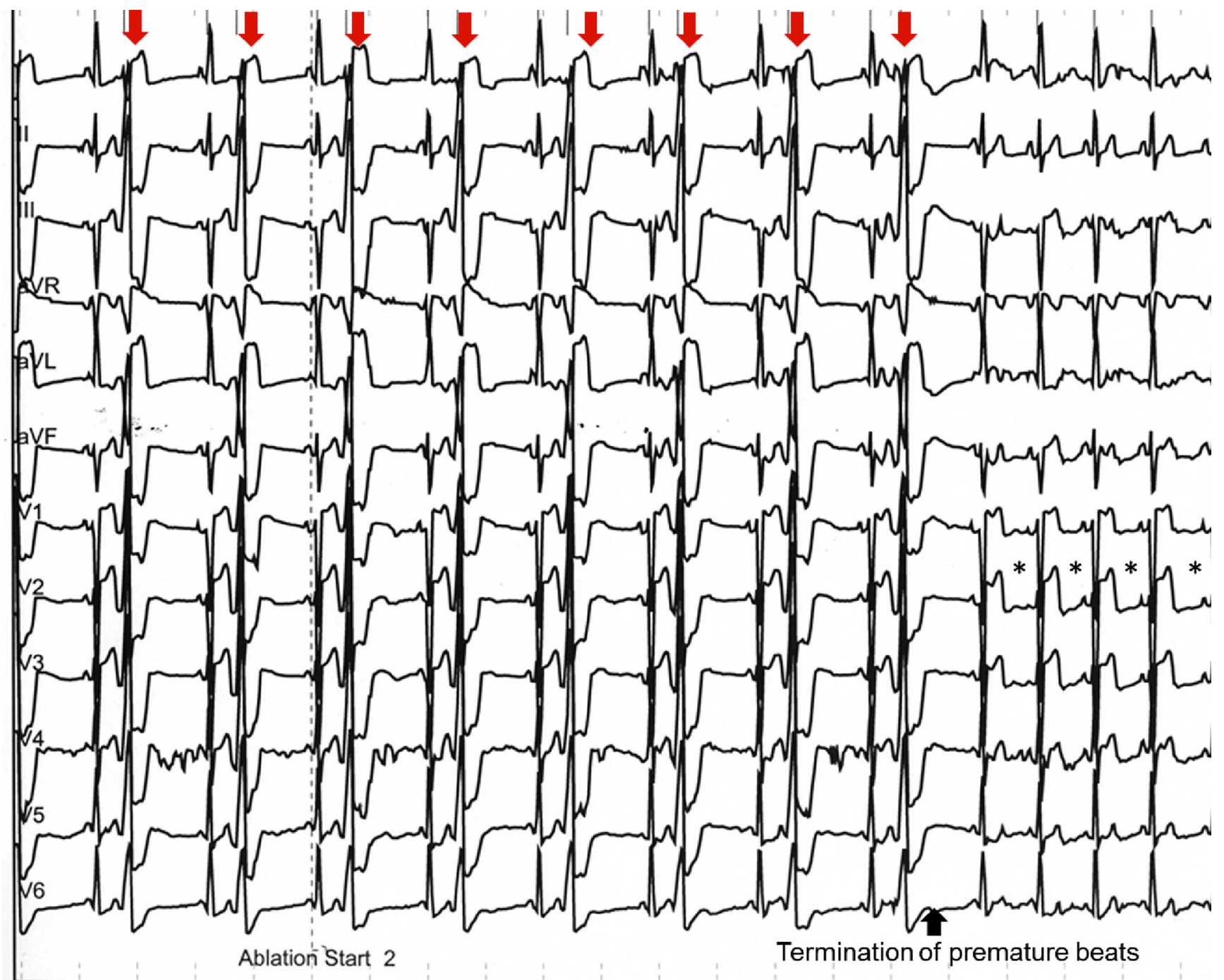

Fig. 3 12-lead ECG with termination of the premature beats (arrow) under radiofrequency ablation. On the right side of the ECG you can see ST elevation $\left(^{*}\right)$ which is documented near the coronary artery. After the ablation, these completely resolved 